\title{
Development of a new software application for supporting research of thermo- mechanical behavior of agri-food and forest products
}

\author{
Jerzy Weres ${ }^{1}$, Wiesław Olek $^{2}$, Sebastian Kujawa ${ }^{3}$, Przemysław Nowak ${ }^{4}$
}

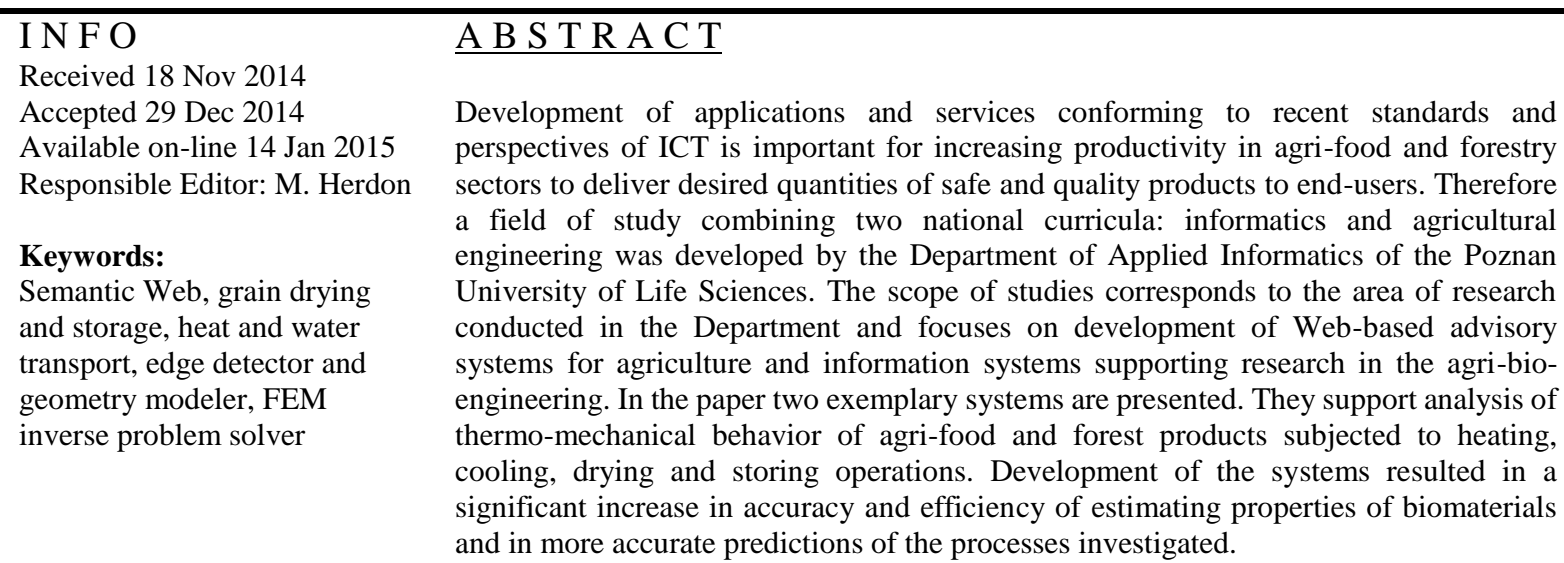

\section{Introduction}

For farmers and industries involved in operations of drying, handling and storage of agricultural products it is essential to have an access to relevant and reliable information to make better decisions and maintain quality standards. To attain it the decision support software should be kept up to date, especially by taking the advantage of recent opportunities of the Future Internet technologies. Such technologies are available for developing Web-based applications and can increase functionality, reliability, usability, maintainability and performance of decision support systems (Weres et al. 2013). Integration of information from diverse Internet sources used to enhance traditional advisory systems, by adoption of the Semantic Web technologies, has been recently a strong trend for future system development (Janjua et al. 2013, Blomqvist 2014). New perspectives for developing Semantic Web-based systems have also been opened by integrating software for traditional platforms and for smartphones (Esposito 2012, McWherter \& Gowell 2012, Weres et al. 2014).

\footnotetext{
${ }^{1}$ Jerzy Weres

Department of Applied Informatics, Institute of Biosystems Engineering, Faculty of Agriculture and Bioengineering, Poznan University of Life Sciences, Poznan, Poland

weres@up.poznan.pl

${ }^{2}$ Wiesław Olek

Department of Mechanical Engineering and Thermal Techniques, Faculty of Wood Technology, Poznan

University of Life Sciences

olek@up.poznan.pl

${ }^{3}$ Sebastian Kujawa

Department of Applied Informatics, Institute of Biosystems Engineering, Faculty of Agriculture and

Bioengineering, Poznan University of Life Sciences, Poznan, Poland

sebastian.kujawa@up.poznan.pl

${ }^{4}$ Przemysław Nowak

Department of Applied Informatics, Institute of Biosystems Engineering, Faculty of Agriculture and Bioengineering, Poznan University of Life Sciences, Poznan, Poland
} 
A serious difficulty appears, however, in performing computer simulations for heat and water transport processes occurring in biomaterials, and this is due to a lack of sufficiently reliable values of material properties. Traditional approaches used to estimate such properties related to shape, size and thermo-mechanical coefficients, necessary to simulate heat and water transport processes, are insufficient for adequate representation of the material behavior by the simulation models. The shape is usually different from the basic 3D solids and the biomaterials are non-homogeneous and often anisotropic. Moreover, the properties are highly dependent on temperature and moisture content.

In earlier works the authors proposed procedures to solve the difficulties (Olek \& Weres 2007, Weres et al. 2009, Olek et al. 2011), but the algorithms required improvement and essential functional integration. They are embedded in measurement of complex 3D object geometry by taking images of object sections, processing the images, detecting required object edges, and mapping the investigated product geometry to a form of the 3D finite element model (Gonzalez \& Woods 2008, Frey \& George 2008, Frączek \& Wróbel 2009, Shih 2010, Weres 2010). Such description of product geometry enhanced quality of mathematical modeling of heat and water transport (Weres 2010, Weres et al. 2014a). The original algorithms proposed to estimate the thermal and mechanical properties of agri-food and forest products were based on the inverse coefficient finite element analysis (Olek \& Weres 2007, Weres et al. 2009, Olek et al. 2011). This approach improved predictions of heat and water transport in investigated biomaterials (Weres et al. 2014b) and was in turn a basis for further enhancements in computer procedures to ensure estimation of sufficiently reliable values of product physical properties.

The objective of the paper was to present results of development of software in cooperation with students of Informatics and Agricultural Engineering curriculum. The two information systems under continual development were selected: the Semantic Web advisory system supporting analysis, design and management of grain crop drying, handling and storage, and the integrated system supporting estimation and analysis of geometric, thermal and diffusive properties of selected agri-food and forest products.

\section{Material and methods}

\subsection{Semantic Web advisory system for grain handling, drying and storage}

The recent version of the Web-based advisory system, designed to support analysis and management of grain crop handling, drying and storage, was constructed according to software engineering standards (Sommerville, 2010). The problem domain was analyzed and documented in the UML 2.4.1 diagrams. The following programming environments and languages were used: Visual Studio 2013, Windows Phone SDK 8, Xamarin, .NET 4.5, ASP .NET 4.5, C++/CLI and C\# 5.0 (Weres et al. 2013).

To provide interoperability and reusability of existing knowledge available on the Web (Kozłowski et al., 2011), the advisory system was embedded in the Semantic Web infrastructure (Janjua et al. 2013, Weres et al. 2013, Blomqvist 2014). Problem domain terms and relations were formally represented by ontologies to facilitate processes of inference essential in analyzing examined properties. The following tools supporting ontology development, storage and visualization were used: the RDF Triple Generator developed by our Department students, AllegroGraph as the triplestore, Gruff as the graphical triplestore browser, and SPARQL as the query language for RDF.

A development of a smartphone light version of the advisory system has also been initiated, and methods appropriate for developing smart apps have been used (Esposito 2012, McWherter \& Gowell 2012, Petzold 2014, Weres et al. 2014). 


\subsection{Integrated system for estimation and analysis of geometric, thermal and diffusive properties of agri-food and forest products}

The integrated system for estimation and analysis of geometric, thermal and diffusive properties of products of biological origin was developed and used to simulate heat and mass transport processes in biomaterials like corn kernels (hybrid variety Pioneer, FAO 280), wheat, segments of carrot roots, pine and beech wood, and wood-based panels. The examined materials were characterized by non-homogeneity and anisotropy, and their shape was irregular. The following properties were subjected to estimation in order to attain reliable values: 3D coordinates of the finite element mesh for representing geometry of a product, thermal conductivity, moisture transport coefficient and convective moisture transfer coefficient.

Product geometry modeling was performed by algorithms of image processing, edge detection for product outer boundaries and inner boundaries between its components, mesh generation for the finite element analysis, and computer visualization of a product and its changing properties (Gonzalez and Woods, 2008, Frey and George, 2008, Frączek and Wróbel, 2009, Shih, 2010, Weres, 2010, Weres et al., 2014a). The existing algorithms were enhanced for accuracy and performance, and integrated with respect to their functionality.

Detailed procedure comprised microtome cutting of a product sample embedded in a synthetic resin, acquisition and processing of digital photographs of sample sections, determination of product outer boundaries and boundaries between its components by one of the edge detection algorithms available, including modified Canny algorithm, design of the finite isoparametric element mesh, and determination of the 3D coordinates for all nodes in the finite element mesh. To visualize a product and changes in its properties a series of algorithms were developed. They were based on the 3D coordinates of the geometry model and comprised the NURBS enhancement, implementation of textures and illumination of a product. For better visualization of changes in product properties the following operations were implemented: moving, rotating, scaling, plane-cutting and time-stepping.

Estimation of sufficiently reliable data on product thermo-mechanical properties was carried out by original algorithms of the inverse finite element modeling. Procedures and experimental set-up for acquiring experimental data for the coefficient inverse problem approach were described in previous papers (Siatkowski et al. 2010, Olek et al. 2011). The algorithms were based on direct and inverse finite element modeling of heat and water transport problems in biomaterials (Weres et al. 2009, Olek et al. 2011, Weres et al. 2014b) and were improved in terms of accuracy, stability and efficiency. They were supplemented with algorithms for constrained local, global and hybrid optimization to minimize the objective function - the difference between values measured experimentally and predicted in simulation (Michalewicz \& Fogel 2004, Nocedal \& Wright 2006, Weres et al. 2009, Siatkowski et al. 2010).

The information system was designed to comprise common components like a graphical user interface, a database, and also integrated subsystems for estimating, predicting, analyzing and visualizing properties of agri-food and forest products during heat and water transport processes. The system was developed according to software engineering standards (Sommerville 2010, Gomaa 2011). The UML 2.4.1 notation, the Visual Paradigm 11.2 diagramming tool, the Visual Studio 2013 programming environment and the C\# v. 5.0 language were used to model and code the software. The Intel Visual Fortran Composer XE for Windows with the IMSL set of mathematical libraries was used to code original finite element algorithms. 


\section{Results}

\subsection{Semantic Web advisory system for grain handling, drying and storage}

Methods described in section 2.1. were implemented to develop a prototype of the advisory system embedded in the Semantic Web infrastructure and thus upgrading the Web-based advisory system "Ziarbit" designed to support analysis and management of grain crop handling, drying and storage (Figure 1).

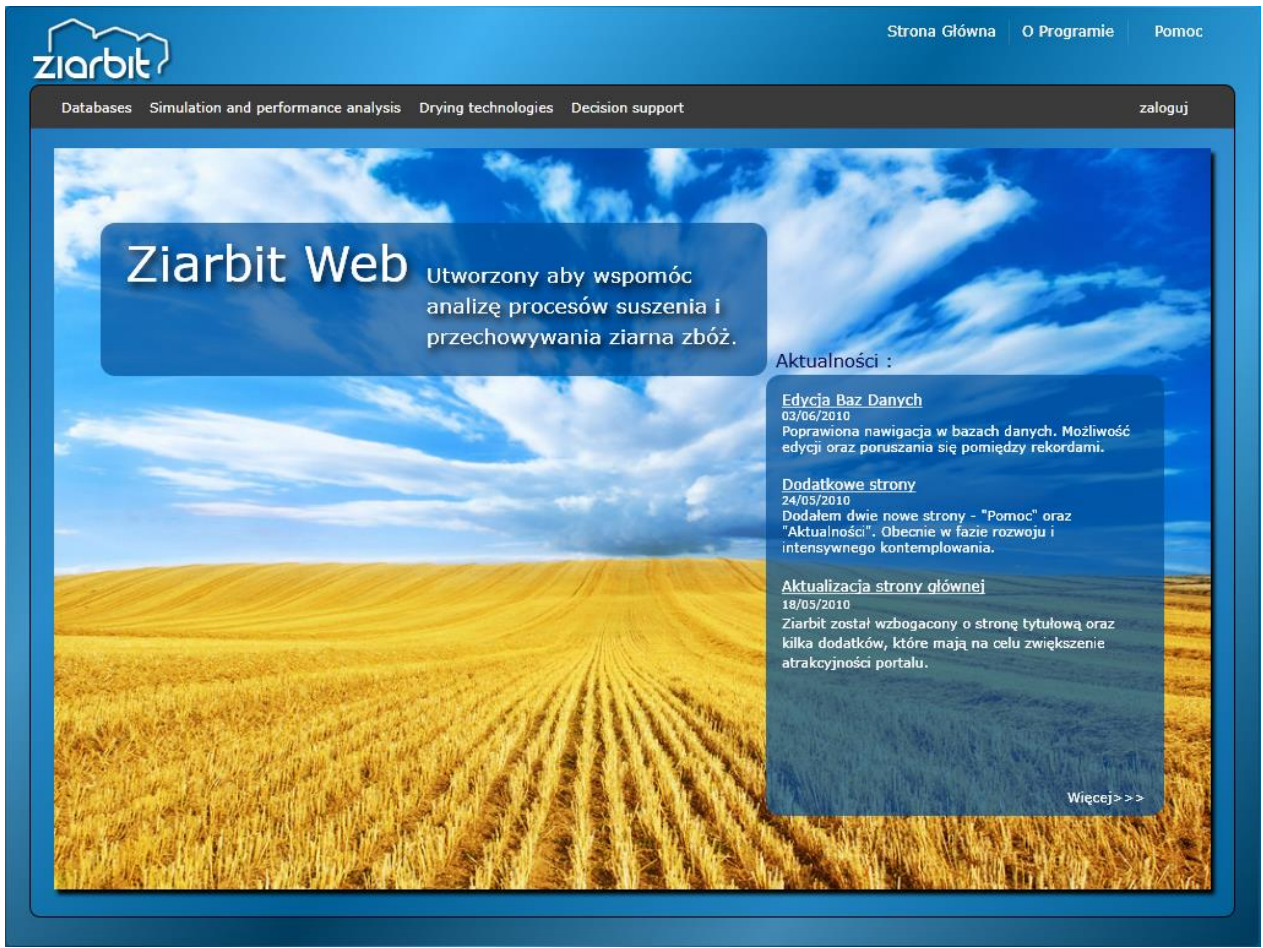

Figure 1. The Web-based advisory system "Ziarbit" supporting analysis and management of grain crop handling, drying and storage.

The RDF Triple Generator was developed to create triples (subject, predicate and object) in the Resource Description Framework for describing Web resources corresponding to the problem domain under consideration (Figure 2).

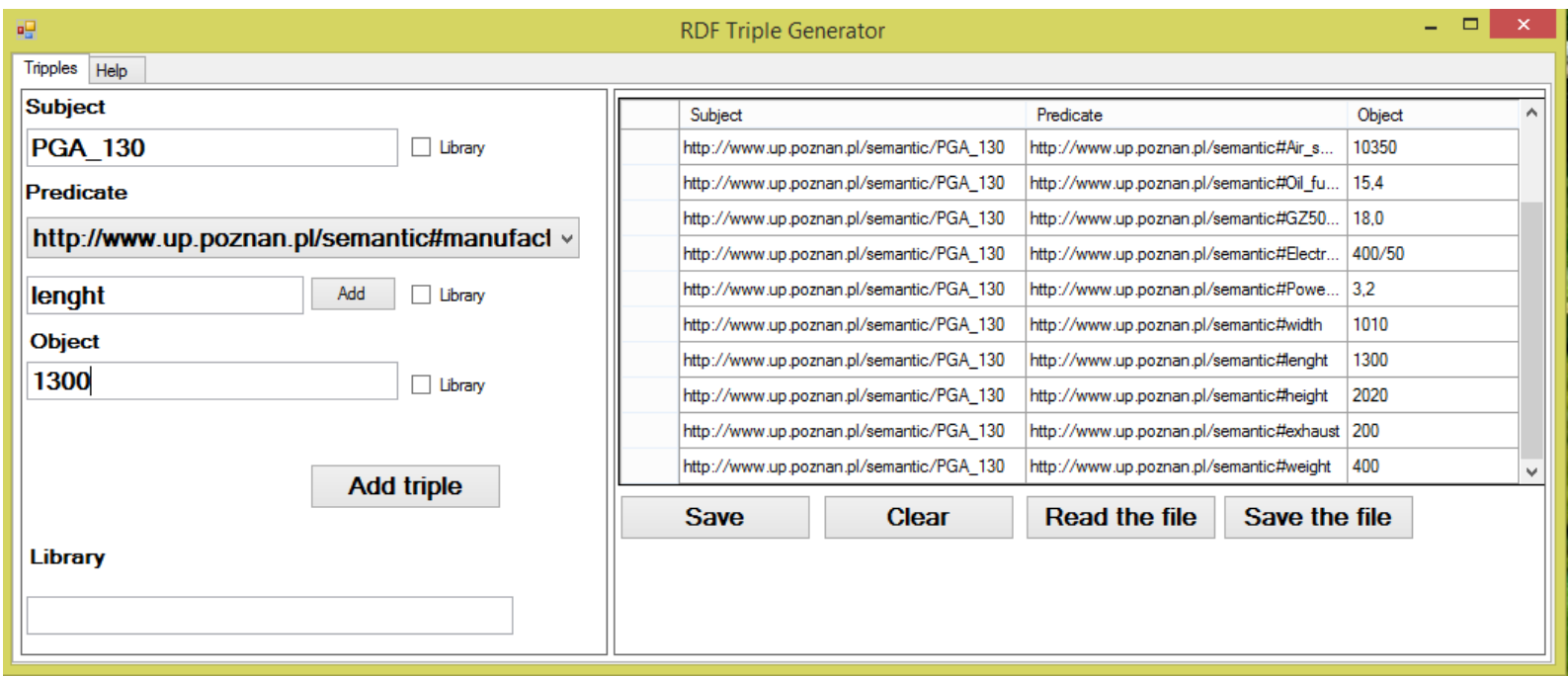

Figure 2. The RDF Triple Generator. 
The Web-based advisory system was enhanced with ontologies. A fragment of the sample graph describing parameters and parts of dryer equipment is depicted in Figure 3.

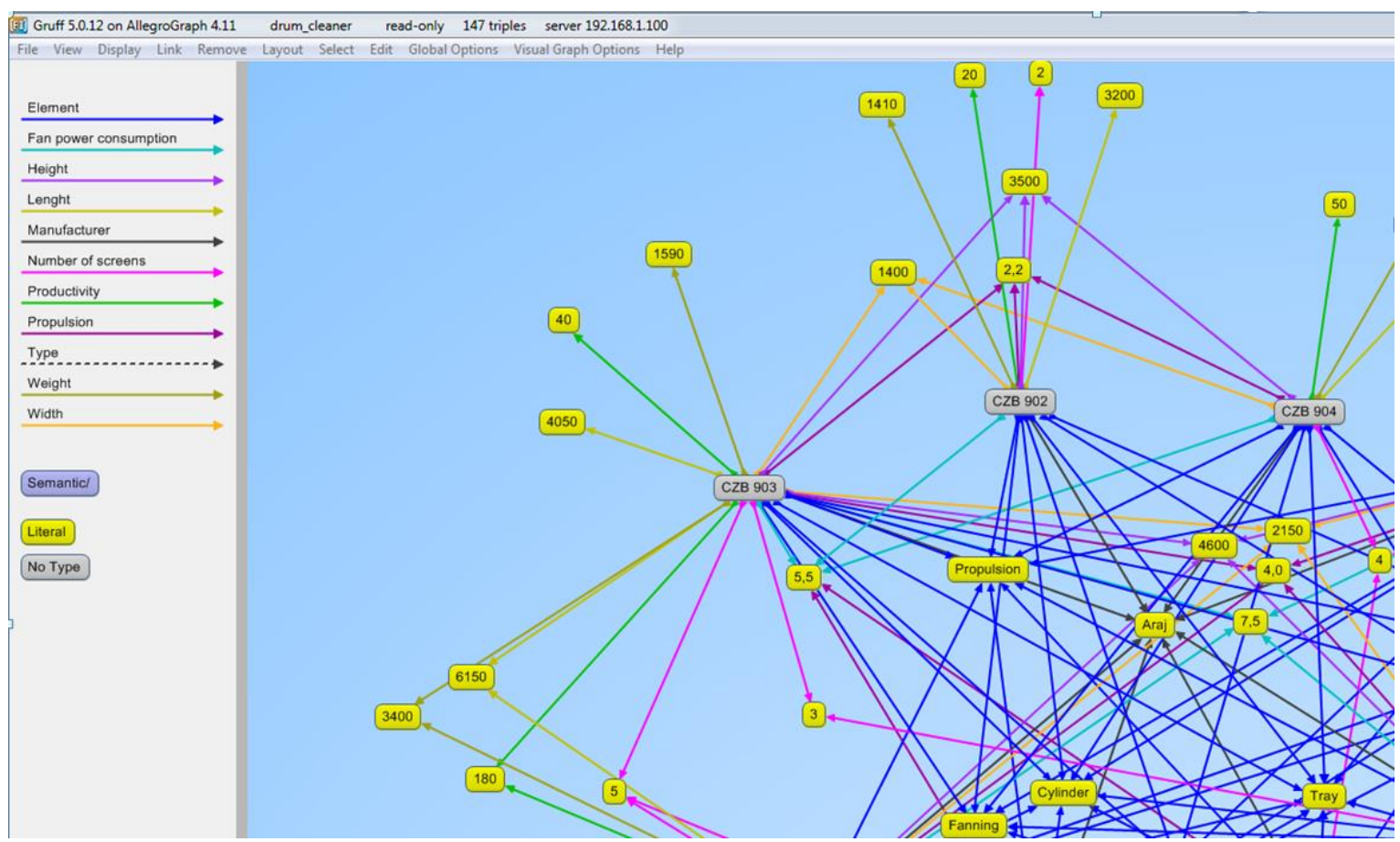

Figure 3. Sample ontology describing parameters and parts of dryer equipment.

Development of a light version of the Semantic Web-based advisory system for smartphones was also initiated (Figure 4).

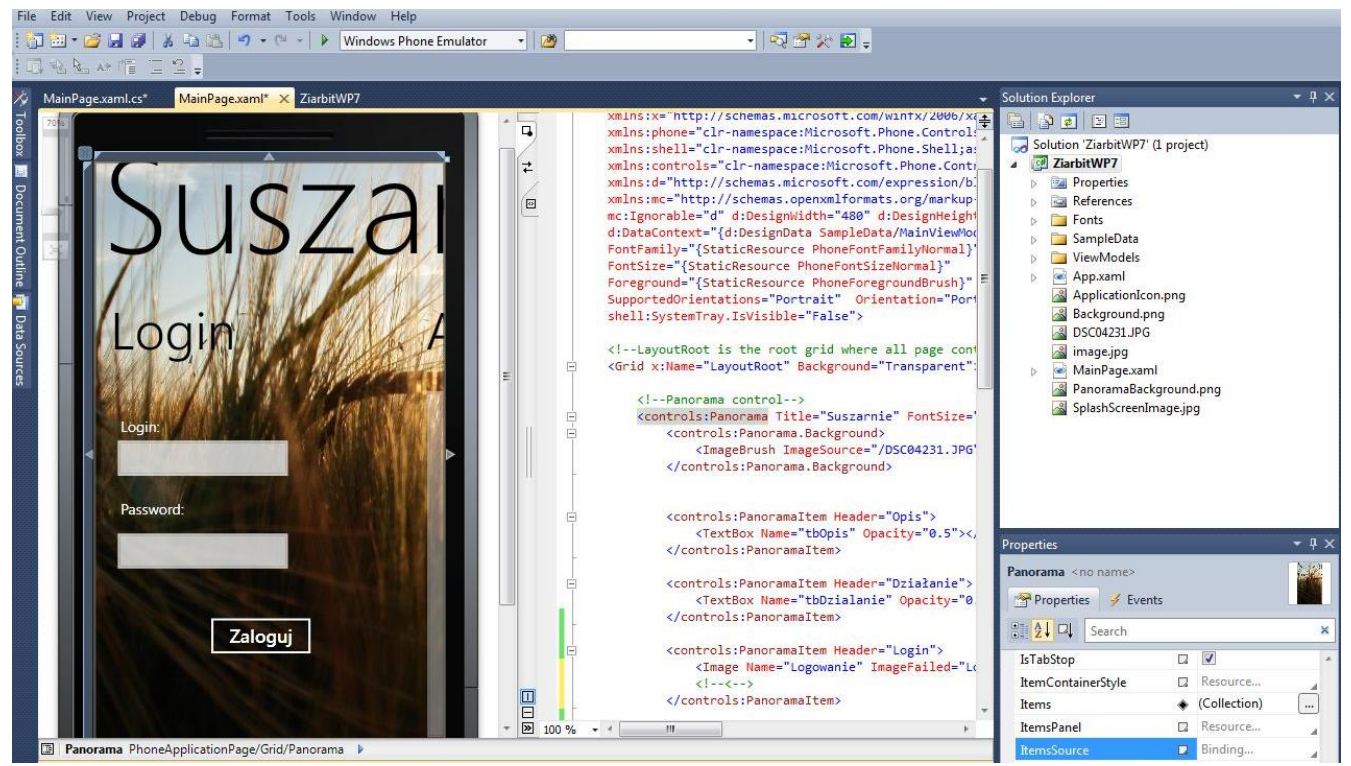

Figure 4. Development of the smartphone app supplementing the Web advisory system.

\subsection{Integrated system for estimation and analysis of geometric, thermal and diffusive properties of agri-food and forest products}

The integrated system was developed according to methods described in section 2.2. The system was used to construct geometrical models of products and to estimate those thermal and diffusive properties for which numerical values are dubious. Next, the system was used to predict temperature and moisture content distributions in time and space for investigated 
products subjected to heat and mass transport, and the estimated property values were used as the input data for simulation models. Quality of estimation of properties was assessed by a procedure in which results of predictions based on estimated data were compared to results obtained in experiments, and it was measured by the global and local relative errors. In the same way the computational accuracy and performance of algorithms were assessed, and the most accurate and efficient procedures were selected to perform all estimations and predictions with the use of the developed information system.

The integrated information system BioProcessSoft was designed as a set of the three following subsystems linked with a user interface and a database:

- 3DMeshNode-3D Mesh Node Generator, 3D geometry data acquisition subsystem for image acquisition and processing, edge detection for object contours and boundaries of internal regions, 3D finite element mesh generation, and collection of coordinates of mesh nodes (Figure 5).

- BioVis - Biomaterial Visualization, 3D geometry modeling and visualization subsystem for presenting products constructed from coordinates of isoparametric finite element mesh nodes in 3D. The nodal coordinates and values of selected product properties in all the nodes and time instants of the investigated process are used as input data. Product geometry can be shown in a selected mode of a wire-frame, solid or a textured model, with enabled or disabled smoothing, illumination and reflection. Affine transformations - translation, rotation and scaling, and removing parts of a product by sectioning appropriate planes are provided to facilitate visualization and analysis of a product and its properties in space and time (Figure 6).

- IPS - Inverse Problem Solver, estimation of thermal and diffusive properties and simulation of heat and water transport processes subsystem. Main functions are: acquisition of experimental data on moisture content and temperature of a product in space and time dimensions; inverse coefficient problem solving based on the finite element analysis of investigated processes and local (trust regions), global (tabu search, simulated annealing and genetic algorithm) and hybrid optimization procedures; direct finite element simulations based on estimated values of product properties; and finallyassessment of quality of predictions, and accuracy and performance of computations (Figure 7). 


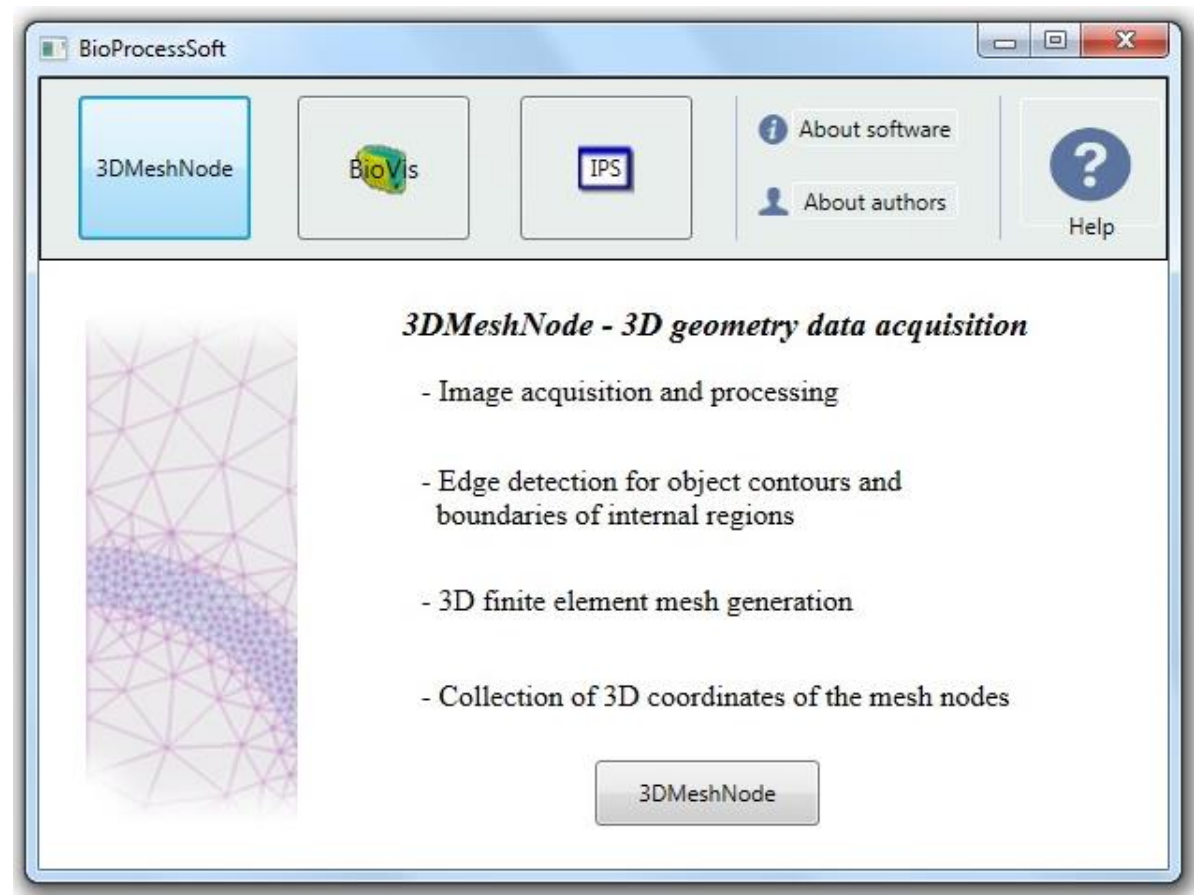

Figure 5. The integrated information system - the 3D geometry data acquisition subsystem.

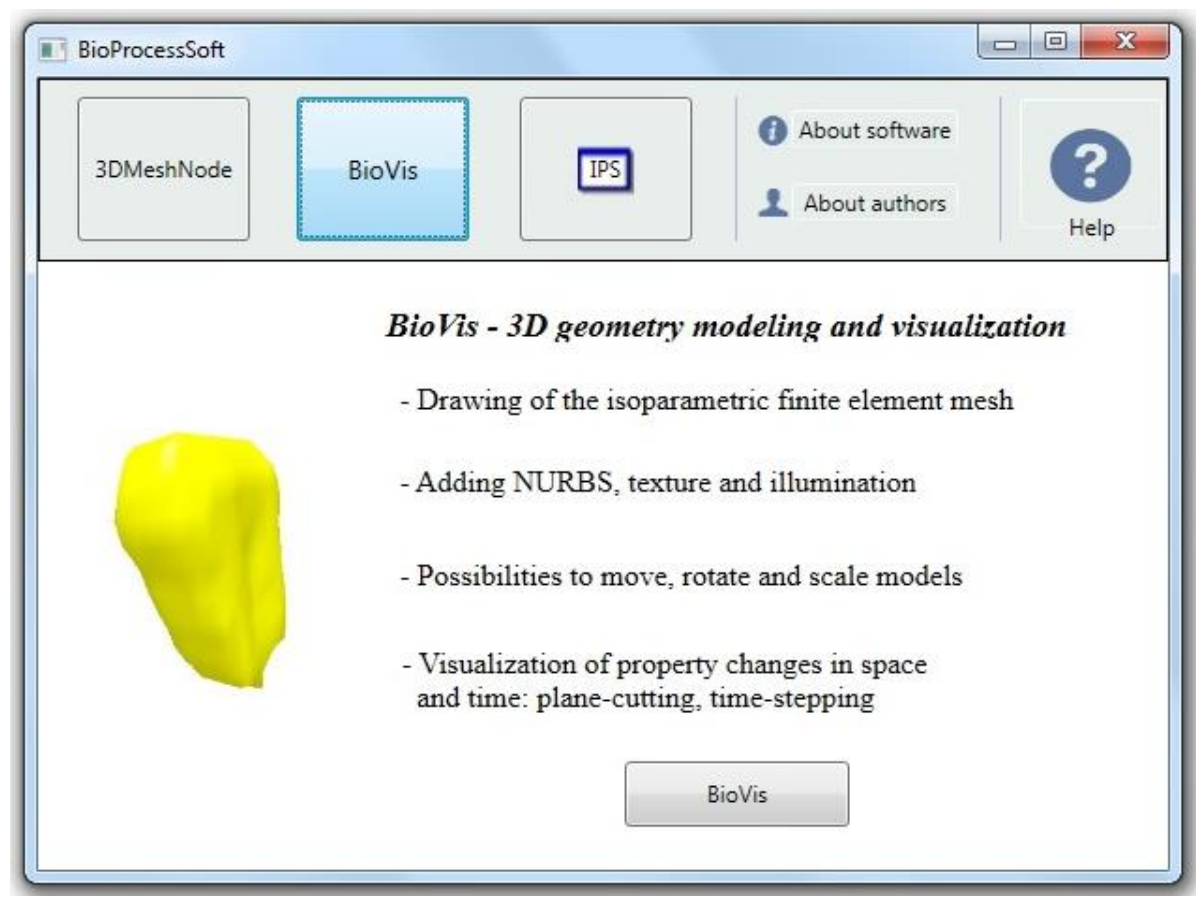

Figure 6. The integrated information system - the 3D geometry modeling and visualization subsystem. 


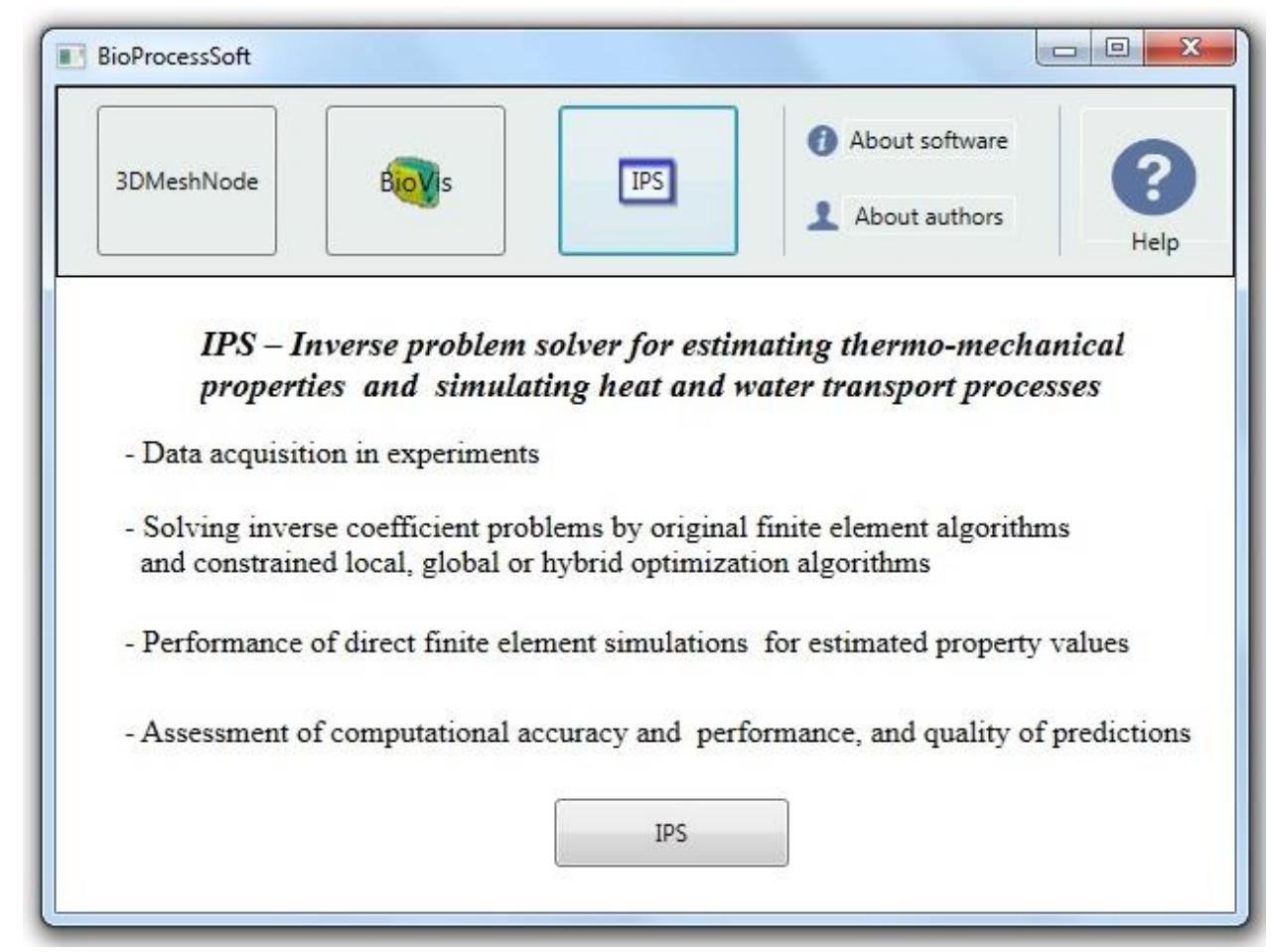

Figure 7. The integrated information system - the estimation of thermal and diffusive properties and simulation of heat and water transport processes subsystem.

Integration and enhancement of procedures for estimating properties of biomaterials, and next for predicting their behavior in heating, cooling and drying operations, resulted in more accurate and effective predictions, exemplified in Figure 8.

The enhancements were due to modifications in the Canny edge detector for finding object boundaries and boundaries between internal product components, improvements in algorithms for automatic construction of the finite element mesh, automatic measuring and storing 3D coordinates of mesh nodes, selection of efficient wrapping procedures for implementing the OpenGL in the .NET environment in C\#, development of more accurate and efficient control procedures for solving inverse problems and for constrained optimization (tabu search, simulated annealing, genetic algorithm, trust regions, and hybrid procedures), and improvement of performance and accuracy comparison for optimization algorithms. Finally, the enhancement was due to adding terms to the convective boundary condition of the water transport mathematical model, and to functions representing estimated coefficients. 


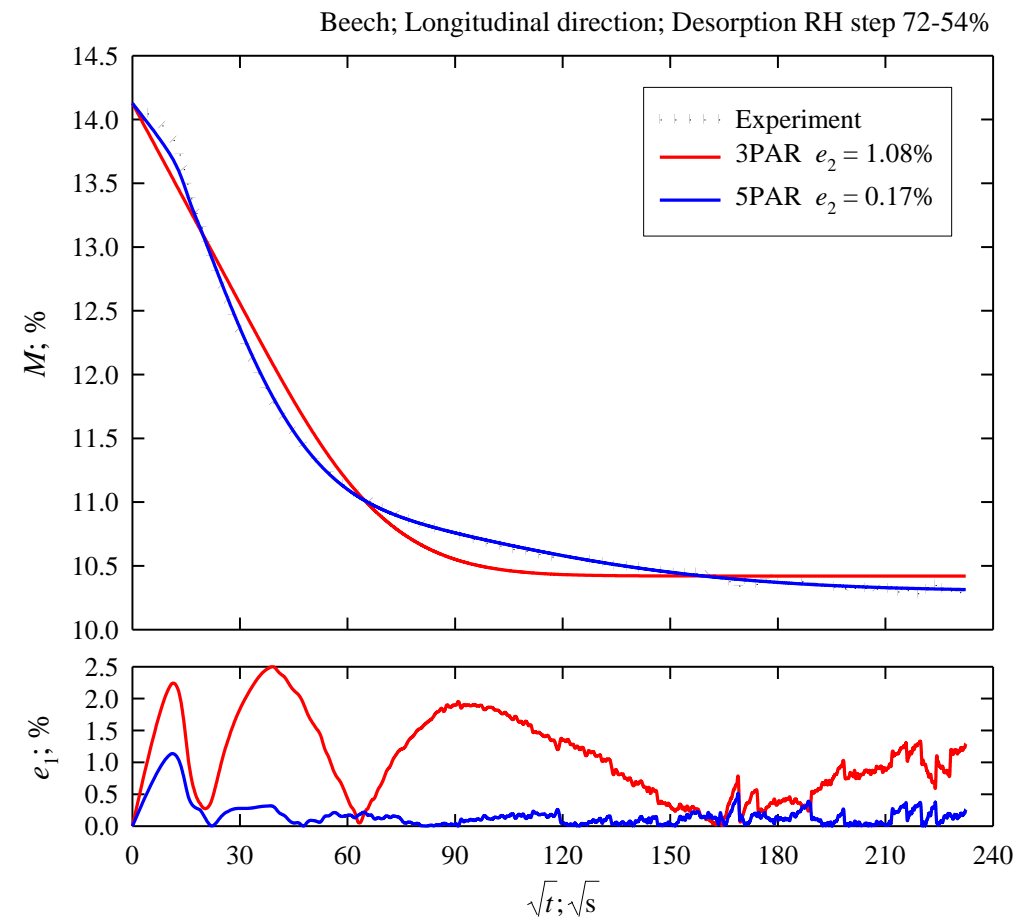

Figure 8. Improvement in accuracy of prediction of the bound water content $(M)$ changes in square root of time $\left(V_{t}\right)$. For the basic 3PAR version of the developed model the global relative error $e_{2}$ was reduced to $1.08 \%$, and for the enhanced version of the boundary condition (5PAR) it was reduced to $0.17 \%$.

\section{Conclusions}

Estimation of geometry and thermo-mechanical properties of agri-food and forest products was improved by modification and integration of original algorithms and software. Prediction of the heat and water transport processes based on the estimated properties was more accurate. The integrated information system manifested satisfactory functionality, usability, effectiveness and efficiency, and the functional requirements were fulfilled. The system offers an essential support in investigating heat and water transport in agri-food and forest products.

\section{Acknowledgments}

The work was financially supported by the National Science Centre as 2011/01/B/NZ9/03169 research grant.

\section{References}

Blomqvist E. 2014. The Use of Semantic Web Technologies for Decision Support - A Survey, Semantic Web Journal, vol. 5, no. 3, pp. 177-201.

Esposito D. 2012. Architecting Mobile Solutions for the Enterprise, Microsoft Press, Redmond, WA, USA.

Frączek J., Wróbel M. 2009. Using computer graphics for 3D reconstruction of seeds (in Polish), Inżynieria Rolnicza, vol. 6, no. 115, 87-94.

Frey P., George P.L. 2008. Mesh Generation, 2nd ed., Wiley-ISTE, Hoboken, NJ, USA. doi: $10.1002 / 9780470611166$

Gomaa H. 2011. Software Modeling and Design: UML, Use Cases, Patterns, and Software Architectures, Cambridge University Press, Cambridge, UK. doi:10.1017/cbo9780511779183 
Gonzalez R. C., Woods R.E. 2008. Digital Image Processing, 3rd ed., Pearson Prentice Hall, Upper Saddle River, NJ, USA.

Janjua N.K., Hussain F.K., Hussain O.K. 2013. Semantic information and knowledge integration through argumentative reasoning to support intelligent decision making, Information Systems Frontiers, vol. 15, no. 2, pp. 167-192. doi:10.1007/s10796-012-9365-x

Kozłowski R.J., Weres J., Rudowicz-Nawrocka J. 2011. Computer decision support systems in farm management (in Polish). AgEngPol Scientific Network, http://www.agengpol.pl/ekspertyzy.aspx.

McWherter J., Gowell S. 2012. Professional Mobile Application Development, Wrox (Wiley), Hoboken, NJ, USA.

Michalewicz Z., Fogel D.B. 2004. How to Solve It: Modern Heuristics, enlarged 2nd ed., Springer, Berlin, DE. doi:10.1007/978-3-662-07807-5

Nocedal, J., Wright S. 2006. Numerical Optimization, 2nd ed., Springer, Berlin, DE.

Olek W., Weres J. 2007. Effects of the method of identification of the diffusion coefficient on accuracy of modeling bound water transfer in wood, Transport in Porous Media, vol. 66, no. 12, 135-144. doi: $10.1007 / 978-1-4020-5480-8 \quad 11$

Olek W., Perré P., Weres J. 2011. Implementation of a relaxation equilibrium term in the convective boundary condition for a better representation of the transient bound water diffusion in wood, Wood Science and Technology, vol. 45, no. 45, 677-691. doi:10.1007/s00226-010-0399-2

Petzold C. 2014. Creating Mobile Apps with Xamarin.Forms, Microsoft Press, Redmond, WA, USA.

Shih F.Y. 2010. Image Processing and Pattern Recognition: Fundamentals and Techniques, Wiley-IEEE Press Hoboken, NJ, USA. doi:10.1002/9780470590416

Siatkowski M., Weres J., Kujawa S. 2010. Comparison of global optimization algorithms in inverse modeling of drying processes of agricultural products (in Polish), Inżynieria Rolnicza, vol. 7, no. 125, 191-198.

Sommerville I. 2010. Software Engineering, 9th ed., Addison Wesley, Upper Saddle River, NJ, USA.

Weres J., Olek W., Kujawa S. 2009. Comparison of optimization algorithms for inverse FEA of heat and mass transport in biomaterials, Journal of Theoretical and Applied Mechanics, vol. 47, no. 3, 701-716.

Weres J. 2010. Information system for acquiring data on geometry of agricultural products exemplified by a corn kernel (in Polish), Inżynieria Rolnicza, vol. 7, no. 125, 229-236.

Weres J., Mueller W., Kluza T., Nowak P., Nowakowski K. 2013. Semantic Web technologies for enhancing Webbased decision support system for designing and managing drying and storage of cereal grains, Journal of Research and Applications in Agricultural Engineering, vol. 58, no. 2, pp. 184-187.

Weres J., Nowak P., Smoczyński M. 2014. A smart app integrated with a Web-based advisory system for designing and managing grain drying and storage, Presentations of International Conference Smart AgriMatics, Pecha Kucha Session: Agri-Food apps, 18-19 June 2014, Paris, France, http://www.smartagrimatics.eu/Portals/4/SAM2014/7\%20Closing\%20session/05\%20Weres.pdf

Weres J., Kiecana M., Balcerzak K. 2014a. Two approaches to representing agri-food product geometry - an original software for constructing finite element models and the 3ds Max approach, Journal of Research and Applications in Agricultural Engineering, vol. 59, no. 1, 155-158.

Weres J., Olek W., Kujawa S., Siatkowski M. 2014b. Integration of software components for determination and analysis of properties of agri-food and forest products, Journal of Research and Applications in Agricultural Engineering, vol. 59, no. 1, 159-163. 